The development of different magnetic states in multisegmented cylindrical nanowires, with alternating magnetic anisotropy, by using the effect of confinement and interaction between segments. 


\title{
Unveiling the Origin of Multi-
}

\section{Domain Structures in}

\section{Compositionally Modulated}

\section{Cylindrical Magnetic Nanowires}

Cristina Bran', Jose Angel Fernandez-Roldan 1,2, Rafael P. Del Real', Agustina Asenjo', Yu-Shen Chen ${ }^{1,3}$, Junli Zhang ${ }^{4}$, Xixiang Zhangt, Arantxa Fraile Rodríguez, ${ }^{5,6}$, Michael Foerster ${ }^{7}$, Lucia Aballe ${ }^{7}$, Oksana Chubykalo-Fesenko ${ }^{1}$ and Manuel Vazquez ${ }^{1}$

1 Instituto de Ciencia de Materiales de Madrid, CSIC, Madrid, 28049, Spain

2 Department of Physics, University of Oviedo, Oviedo, 33007, Spain

${ }^{3}$ Department of Chemical Engineering and Materials Science, Yuan-Ze University, Chung-Li 32003, Taiwan

${ }^{4}$ Physical Science and Engineering Division (PSE), King Abdullah University of Science and Technology (KAUST), Thuwal 23955, Saudi Arabia

${ }^{5}$ Departament de Física de la Matèria Condensada, Universitat de Barcelona, Barcelona, 08028, Spain

${ }^{6}$ Institut de Nanociencia i Nanotecnologia (IN2UB), Universitat de Barcelona, Barcelona, 08028, Spain

${ }^{7}$ ALBA Synchrotron Light Facility, CELLS, Barcelona, 08290, Spain

\begin{abstract}
CoNi/Ni multisegmented cylindrical nanowires were synthesized via electrochemical route. The wires are $140 \mathrm{~nm}$ in diameter, with $1000 \mathrm{~nm}$ long $\mathrm{Ni}$ segments, and CoNi segments between 600 and $1400 \mathrm{~nm}$ in length. The magnetic configuration was imaged by XMCD-PEEM in the demagnetized state and at remanence after magnetizing axially and perpendicularly. Ni segments, with cubic crystal symmetry, show an axial magnetic
\end{abstract}


configuration with a small curling component at the surface. In turn, CoNi segments, with hexagonal crystal symmetry and a strong magnetocrystalline anisotropy perpendicular to the nanowires, show a single vortex state in the shorter segments and multi-vortex or multi-transverse magnetic configurations in medium and long segments, respectively. A detailed study by micromagnetic simulations reveals that the magnetic configuration is determined mainly by the coupling between soft $\mathrm{Ni}$ and harder CoNi segments. For short CoNi segments, Ni segments are magnetostatically coupled and the chirality of the single vortex formed in CoNi remains the same as that of the curling in neighbouring $\mathrm{Ni}$ segments. For longer CoNi segments, the remanent state is either multi-vortex or multitransverse state depending on whether the previously applied field was parallel or perpendicular to the magnetocrystalline axis. The results point out the relevance of the cylindrical geometry to promote the occurrence of complex magneto-chiral effects and provide key information for the design of cylindrical magnetic nanowires for multiple applications.

Keywords: cylindrical magnetic nanowires; magnetic domains; vortex structures; XMCDPEEM; micromagnetic modelling, magnetocrystalline anisotropy.

Cylindrical magnetic nanowires (NWs) are one of the most promising nanostructures studied in the last years where the curvature is in the origin of novel magnetic applications. ${ }^{1}$ They are proposed as future materials for three-dimensional (3D) information technologies such as magnetic recording, spincaloritronics, shift registers and logic gates, as well as in sensing architectures or multiple biomedical applications. The use of 3D magnetic structures for memory and sensing application will require "information carriers", a role to be played by 3D domain walls (DWs) and spin waves. ${ }^{2-5}$ The emerging interest toward cylindrical magnetic NWs is related to the fact that their geometry promotes topologically non-trivial 3D magnetic structures such as Bloch-point domain walls, ${ }^{3}$ complex configurations that connect vortex domains with opposite chirality, vortices or skyrmion tubes. ${ }^{4}$ While domain walls and their dynamics are well explored in lithographed planar nanostructures, 3D domain walls in cylindrical NWs are 
largely unexplored. Vortex (Bloch point) domain walls present magnetization dynamics different from that of standard transverse walls, where the left-handed and right-handed walls exhibit different velocities due to an inversion symmetry breaking. ${ }^{6}$ Furthermore, the Walker breakdown which limits the domain wall propagation in flat geometries (nanostrips), is absent in cylindrical NWs. ${ }^{7}$

The design of cylindrical NWs for specific applications requires understanding and fully controlling the magnetic configurations of individual magnetic NWs, determined by the composition through the balance between the geometrical shape and magnetocrystalline anisotropy. Importantly, cylindrical NWs can present multifunctional behavior due to their interconnected response to electric and magnetic fields, electric current, mechanical stresses or thermal gradients. ${ }^{8}$ The tailoring of magnetic properties, by fabricating either homogeneous NWs or multilayers combining different materials and engineering the NW geometry (e.g. producing bamboo-like structures with modulations in diameter or notches along their lengths) could provide a rich playground for functionalization. ${ }^{9-12}$

The control and stabilization of domains and domain walls in cylindrical NWs has been achieved in multilayer/multisegmented structures ${ }^{13-18}$ by alternating magnetic segments of different magnetic behavior or with non-magnetic metallic segments. This design can provide active channels for domain wall pinning or spin wave manipulation. The multilayers formed by non-magnetic/magnetic layers can be used for domain wall pinning or confinement of specific domains. They are employed in a number of technologies ${ }^{19}$ and are promising candidates for spin transfer nano-oscillators with ultra-wide operating microwave frequency with a synchronization possibility via magnetostatic coupling $20-23,14$ or as shift-registers. ${ }^{24}$ Recently, the magnetization reversal following a ratchet effect was observed in $\mathrm{FeCo} / \mathrm{Cu}$ multisegmented NWs. The observed unidirectional propagation, irrespective of the field direction, is interpreted as originating in the broken symmetry induced by the tailored length of $\mathrm{FeCo}$ segments, promoted by the magnetostatic coupling between adjacent segments. ${ }^{15}$ On the other hand, the magnetic/magnetic multisegmented NWs offer the possibility to produce a system with alternating anisotropies which can pin domain walls between the segments ${ }^{18,25,26}$ or nucleate and stabilize them due to the influence of the neighboring material. 
In this context, Co-based alloy NWs are good candidates for tailoring designed magnetic anisotropies and consequently, magnetic behavior. The low magnetocrystalline anisotropy of cubic, $b c c$ and $f c c$ symmetry, materials (Fe, Ni, NiFe, FeCo) combined with the high anisotropy of the hexagonal hcp symmetry of Co allows tailoring the magnetic properties of the system. In the particular case of NWs with low magnetocrystaline anisotropy, the shape determines the formation of single axially magnetized domain state. In contrast, in Co NWs the magnetocrystalline anisotropy plays a decisive role as they crystallize usually into a $h c p$ phase with a strong magnetocrystalline anisotropy energy constant and an easy axis (the $c$-axis) in most cases nearly perpendicular to the NWs. ${ }^{27,28}$ This promotes the formation of vortex, transverse and more exotic combined magnetic configurations. ${ }^{29}$ While multisegmented NWs with alternating magnetic anisotropy have been studied in several occasions, ${ }^{13,18,25,26,30}$ there is no report to our knowledge of the detailed mechanism or the origin for the formation of specific domains or magnetic configurations.

CoNi NWs were shown to present hybrid magnetic states consisting of a mixture of perpendicular and vortex domains. ${ }^{31}$ In the present work we prepared multisegmented CoNi/Ni NWs with tailored alternating magnetic anisotropy with the aim to control the occurrence of different states by using the effect of confinement and interaction between segments. Various magnetic states (e.g. demagnetized state and at remanence after applied field in different orientations) have been experimentally determined by X-ray Magnetic Circular Dichroism combined with Photoemission Electron Microscopy (XMCDPEEM). Complementary micromagnetic simulations helped us to unveil the mechanism that determines the formation of different domain structures observed in CoNi segments.

\section{Results and Discussions}

Three types of [CoNi/Ni $]_{10} \mathrm{NWs}$ (with constant $\mathrm{Ni}$ length and different CoNi segment lengths), were prepared by electroplating filling the pores of anodic aluminium oxide (AAO) membranes [For details, see the Supplementary Information. ${ }^{25,31,32}$ ]

The High-Resolution Transmission Electron Microscopy (HRTEM) analysis confirms the multisegmented morphology with a uniform diameter, and periodic and sharp interfaces. Quantitative analysis reveals that the NWs diameter is $\approx 140 \mathrm{~nm}$, with lengths of $600 \mathrm{~nm}$, 
$1200 \mathrm{~nm}$ and $1400 \mathrm{~nm}$ for CoNi and about $1000 \mathrm{~nm}$ for Ni segments (Figures 1 (a), (b)). The Energy-Dispersive X-ray Spectroscopy (XEDS) measurement of CoNi segments indicates an 85:15 atom ratio of Co: $\mathrm{Ni}$, i.e. a $\mathrm{Co}_{85} \mathrm{Ni}_{15} / \mathrm{Ni}$ composition for the multisegmented NWs. XEDS profile shows sharp interfaces between the segments, with an intermixing area of around $20 \mathrm{~nm}$ for Co into the Ni layer (Figure 1(c)).

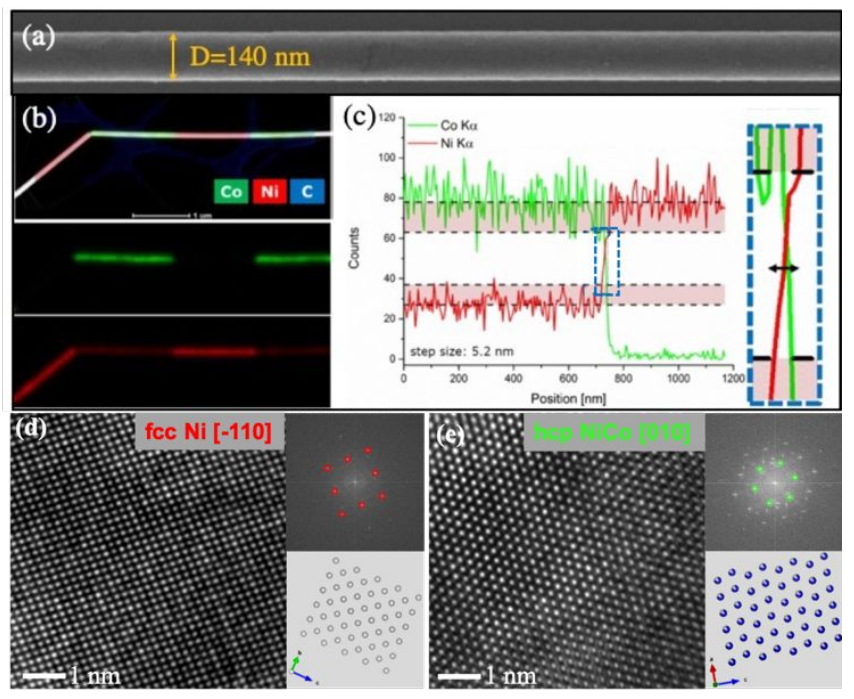

Figure 1. (a) SEM image of a single CoNi $(1200 \mathrm{~nm}) / \mathrm{Ni}(1000 \mathrm{~nm}) \mathrm{NW}$, (b) Scanning Transmission Electron Microscopy (STEM) based on core-loss electron energy loss spectroscopy, XEDS chemical mapping of CoNi $(1400 \mathrm{~nm}) / \mathrm{Ni}(1000 \mathrm{~nm})$ and (c) XEDS profile along the interface between layers, (d)-(e) atomic-resolved HRTEM images and corresponding FFT patterns (topright panel) and atomic model (bottom-right panel).

Figure 1(d) shows a typical atomic HRTEM image in Ni segments, which corresponds to the [-110] crystallographic orientation of face-centred cubic (fcc) Ni. The Fast Fourier Transform (FFT) patterns in the top-right panel and the atomic model in bottom-right panel of Fig. 1 (d) show the reciprocal and real space of the crystal structure and the crystal orientation of fcc $\mathrm{Ni}$, agreeing well with the HRTEM results. The crystallographic structure of $\mathrm{Co}_{85} \mathrm{Ni}_{15}$ segment was also verified by the HRTEM (Fig. 1 (e)) and the corresponding FFT pattern in Figure 1 (e)-top-right panel, showing a hcp crystalline structure oriented along [010] direction.

Magnetic imaging was performed by XMCD-PEEM. This technique has the capability to explore the magnetization configuration of individual cylindrical NWs providing simultaneous information of the magnetization distribution at the surface (direct emitted photoemission from the wire) and inside the NWs (photoemission from the substrate 
from photons transmitted through the wire). ${ }^{11,33-36}$ The measurements were taken at both Co and $\mathrm{Ni}-\mathrm{L}_{3}$ edges (778 and $852 \mathrm{eV}$, respectively) to get a full picture of the magnetic structure along the multisegmented individual NWs. Prior to the experiments the samples were demagnetized in a decreasing-amplitude alternating magnetic field perpendicular to the NW. XMCD images present different magnetic contrast when the X-ray beam incidence is perpendicular to the NW axis: (i) bright or (ii) dark contrast indicate that the magnetic moments are oriented parallel or anti-parallel to the X-ray propagation vector, respectively, and (iii) grey contrast when the magnetic moments are oriented perpendicular to the X-rays, i.e. parallel to the NW axis.

Figure 2 depicts the XMCD-PEEM images taken at the Co-edge, of CoNi/Ni multisegmented NWs with lengths of the CoNi segments of $600 \mathrm{~nm}$ (Figure 2(a)), 1200 nm (Figure 2(b)), and $1400 \mathrm{~nm}$ (Figure 2(c)). For the NW with shorter CoNi segments the data (both the direct signal and the signal from the shadow ${ }^{11}$ ) show that each CoNi segment presents a single vortex state (either bright or dark contrast, depending on the chirality). When the upper part of the vortex domain is oriented parallel to the x-ray propagation vector, the resulting contrast on the wire appears bright (i.e. dark in the shadow), while for the antiparallel orientation the resulting contrast appears dark/bright on the wire and shadow, respectively. For a more detailed description of the magnetic contrast formation, see Supplementary Information. In the CoNi with intermediate length up to three vortices with alternating chirality (contrast) are formed inside each segment (Figure 2(b)). Finally, in the NWs with longest CoNi segments $(1400 \mathrm{~nm})$, we observe the occurrence of either vortices or periodically transversal domains (Figure $2\left(c_{1}-C_{2}\right)$ ). Figure $2\left(c_{1}\right)$ shows a nanowire presenting one or two vortices in the CoNi segments. A second $\mathrm{CoNi} / \mathrm{Ni}$ nanowire is presented in Fig. $2\left(\mathrm{C}_{2}\right)$ showing a combination of vortices and transversal domains.

A close-up image of the marked segment (red dashed square) in Fig. $2\left(c_{2}\right)$ is shown in Fig. $2\left(d_{1}\right)$. At the left side of the CoNi segment first a vortex domain with a length of about $250 \mathrm{~nm}$ is formed followed by the periodic transversal domains. The width of the transverse domains is estimated from the XMCD contrast profile across the wire (at the position of green dashed line) to be of about $105 \mathrm{~nm}$ (Figure $\left.2\left(\mathrm{~d}_{2}\right)\right)$. 


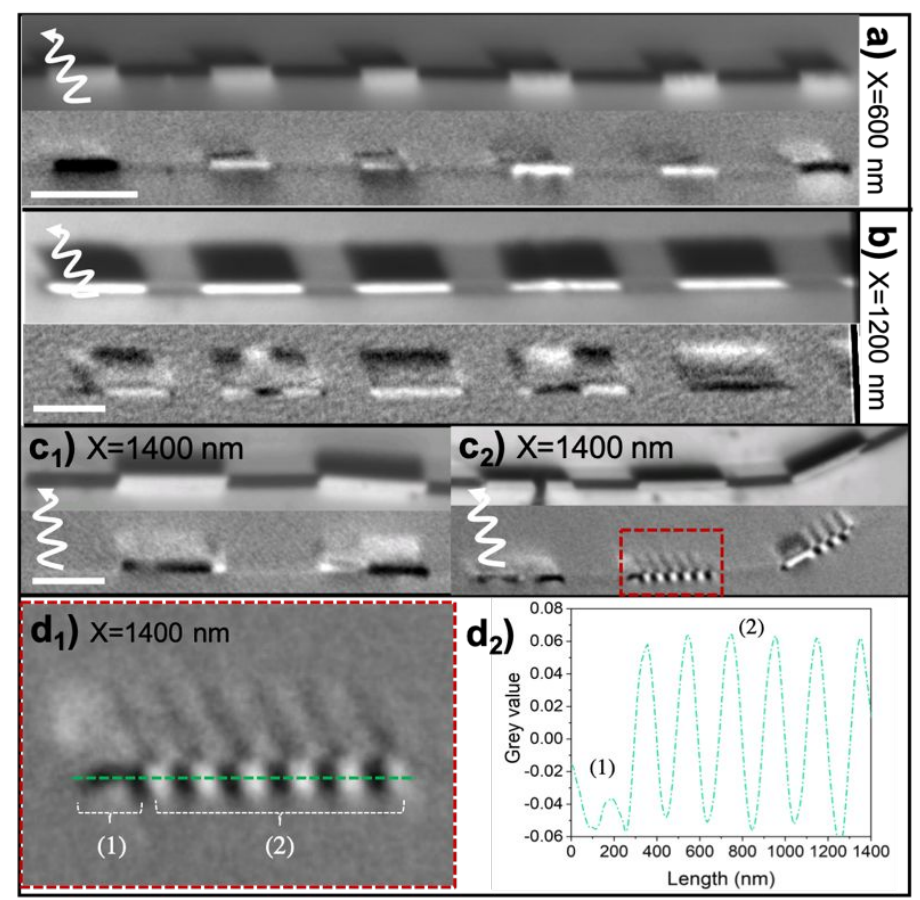

Figure 2. Chemical contrast (upper images) and XMCD-PEEM (lower images) contrast at $\mathrm{Co}_{3_{3}}{ }^{-}$ edge of $\mathrm{CoNi}(\mathrm{x}) / \mathrm{Ni}(1000 \mathrm{~nm})$ multisegmented NWs with (a) $\mathrm{x}=600 \mathrm{~nm},(\mathrm{~b}) \mathrm{x}=1200 \mathrm{~nm}$ and (c) $x=1400 \mathrm{~nm} .\left(d_{1}\right)$ XMCD-PEEM image of transversal domains in a $1400 \mathrm{~nm}$ CoNi segment, $\left(d_{2}\right)$ XMCD profile of the CoNi segment $\left(d_{1}\right)$ presented at the position marked by dashed horizontal green line. Scale bar $1 \mu \mathrm{m}$.

To summarize, the experimental results reveal the presence of one to three vortex domains and a transversal multi-domain configuration in CoNi segments depending on their length, while Ni segments are in single axial domain state (not shown here).

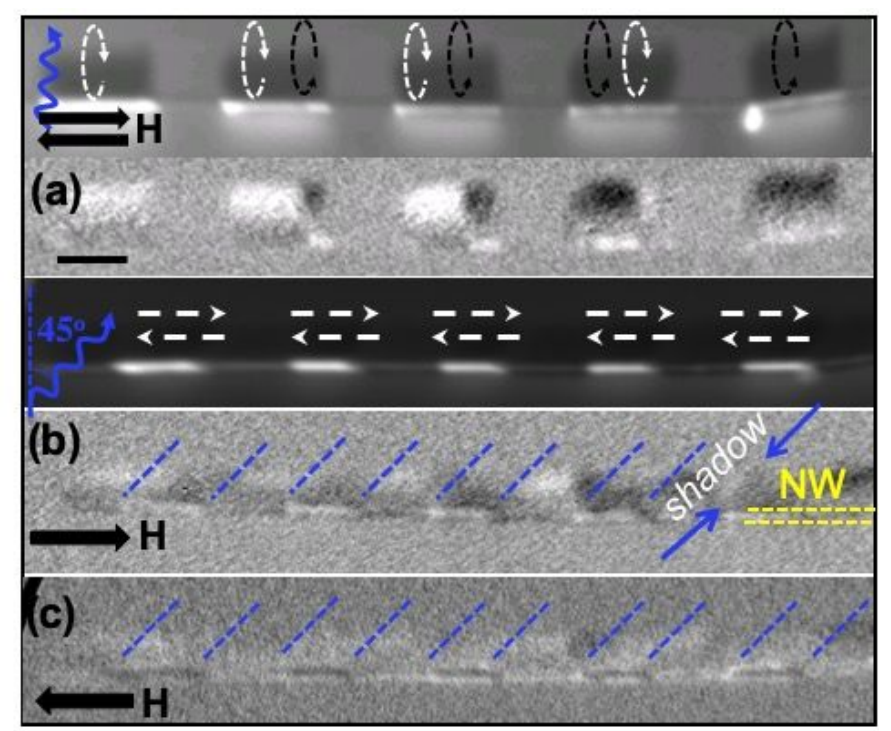

Figure 3. Chemical contrast (upper images) and XMCD-PEEM (lower images) measurements of a CoNi $(x) / N i N W$ with $x=1200 \mathrm{~nm}$. XMCD-PEEM images taken (a) at $C_{0} L_{3}$-edge with the beam 
pointing perpendicular to the NW axis, (b) and (c) at $\mathrm{Ni}_{3}$-edge with the $\mathrm{X}$-ray beam pointing at $45^{\circ}$ under $\pm 170 \mathrm{mT}$ magnetic fields as indicated by the solid black arrows. The dotted blue lines in (b) and (c) delimitate the shadow of Ni segments produced by the beam oriented at $45^{\circ}$. The scale bar is $1 \mu \mathrm{m}$.

To study the evolution of magnetic configurations under applied field, we placed CoNi $(1200 \mathrm{~nm}) / \mathrm{Ni}(1000 \mathrm{~nm}) \mathrm{NW}$ in a sample holder enabling in-situ application of in-plane magnetic fields up to $\pm 170 \mathrm{mT} .{ }^{37}$ The magnetic field is applied along the nanowire axis as indicated by the black arrows in Figure 3. Due to the difficulty to obtain XMCD images with the required spatial resolution under applied field, which disturbs the low energy electrons in the PEEM, we restricted our observations to the remanent states, i.e. after reducing the field back to zero. Figure $3(\mathrm{a})$ shows the magnetic configuration of $\mathrm{CoNi}$ segments after magnetic fields were applied along the nanowire axis in both senses and various strengths: the magnetic state of CoNi segments (single or double vortices with opposite chirality) stays unchanged, denoting a stable minimum-energy configuration, likely stabilized by the large perpendicular magnetocrystalline anisotropy of Co (Figure 3(a)). In order to detect the magnetization of both CoNi and Ni segments (with magnetic moment oriented perpendicular and parallel to the NW axis, respectively), the sample was rotated to 45 degrees with respect to the beam incidence and the photon energy was swept between the $\mathrm{Co}$ and $\mathrm{Ni}_{3}$-edges. The magnetization of the $\mathrm{Ni}$ segments lies along the nanowire axis and switches the orientation with the magnetic field. Figure 3(b)

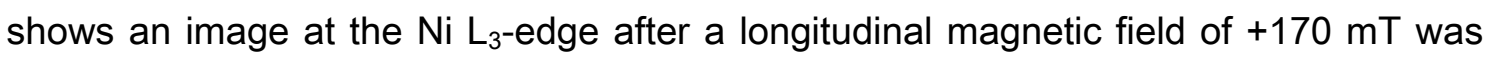
applied. A uniform bright contrast is observed, suggesting a single magnetization state oriented along the X-ray propagation vector. A weak bright and dark contrast is observed also in the CoNi layers, even if the $\mathrm{Ni}$ content is only $15 \%$. By changing the magnetic field polarity to $-170 \mathrm{mT}$, the magnetization in Ni segments is reversed (uniform dark contrast along the NW in the XMCD-PEEM image of Figure 3(c)). Although images in Fig. 3(b) and particularly (c) are of lower quality due to technical issues during the synchrotron measurements, the contrast and resolution are enough to identify the magnetization direction in both types of segments. To sum up, under maximum available applied field, Ni segments reverse axial magnetization while vortex domains in CoNi segments remain unchanged, confirming their respective soft and hard magnetic character. 
To reveal the origin of different magnetic structures observed in $\mathrm{Ni}$ and, most interestingly, in CoNi segments, micromagnetic simulations have been performed. In principle, several factors can influence the magnetic structure in CoNi: (i) the segment length through both, the confinement and the magnetostatic shape anisotropy effects, (ii) the magnetization direction of the neighbouring Ni segments through stray fields, and (iii) the CoNi hcp phase anisotropy direction with respect to the applied field.

In order to disentangle the importance of different contributions we have performed micromagnetic simulations with the finite difference micromagnetic software mumax $3,{ }^{38}$ with a maximum discretization size of $1.5 \mathrm{~nm}$. Periodic boundary conditions along the NW axis direction mirror the experimental conditions of NWs with large number of segments, and remove nucleation effects at the ends of otherwise shorter simulated NWs. The diameter of the NW has been set to $140 \mathrm{~nm}$, the length of Ni segment to 900 $\mathrm{nm}$ while the length of the CoNi segments was varied. The material parameters, crystal structure, and geometrical parameters of the segments are collected in Table I. Following the XMCD-PEEM observations, the remanent states of multisegmented CoNi/Ni NWs have been evaluated for different lengths of CoNi segments and an external magnetic field previously applied perpendicular to the NW axis, i.e. either parallel or perpendicular to the easy magnetization axis of the $h c p$ structure.

Table I. Parameters used in micromagnetic modelling: saturation magnetization, $\mu_{0} M_{s}$, exchange stiffness, $A_{e x}$, crystalline structure, easy axis direction, magnetocrystalline anisotropy constant, $\mathrm{K}_{1}$, and length of CoNi and $\mathrm{Ni}$ segments. Saturation magnetization and exchange stiffness for CoNi alloys have been obtained by linear interpolation with the Co content of the alloy in Ref. ${ }^{31}$

\begin{tabular}{|c|c|c|c|c|c|c|}
\hline Material & $\begin{array}{c}\boldsymbol{\mu}_{\mathbf{0}} \mathbf{M}_{\mathbf{s}} \\
(\mathrm{T})\end{array}$ & $\begin{array}{c}\mathbf{A}_{\mathbf{e x}} \\
(\mathrm{pJ} / \mathrm{m})\end{array}$ & $\begin{array}{c}\text { Crystal } \\
\text { structure }\end{array}$ & Easy axis & $\begin{array}{c}\mathbf{K}_{\mathbf{1}} \\
\left(\mathrm{kJm}^{-3}\right)\end{array}$ & $\begin{array}{c}\text { Segment length } \\
(\mathrm{nm})\end{array}$ \\
\hline $\begin{array}{c}\mathrm{Ni}(111) \\
{\left[{ }^{27}\right]}\end{array}$ & 0.61 & 3.4 & $f c c$ & $\begin{array}{c}{[111] \| \mathrm{NW}} \\
\text { axis }\end{array}$ & -4.8 & 900 \\
\hline $\begin{array}{c}\mathrm{Co}_{85} \mathrm{Ni}_{15} \\
{[13,39,40]}\end{array}$ & 1.60 & 26.0 & $h c p$ & $\begin{array}{c}\text { At } 88^{\circ} \text { with } \\
\text { NW axis }\end{array}$ & 350 & $600,900,1200$ \\
\hline
\end{tabular}

The magnetic configurations in Figures 4(a)-(c) correspond to remanent states obtained after magnetizing the nanowire under $2 \mathrm{~T}$ magnetic field applied in the $\mathrm{y}$-direction, i.e perpendicular to the NW (x-direction) and to the hcp anisotropy easy axis (z-direction). In order to analyze the curling component, $\mathrm{m}_{\mathrm{y}}$ component of the magnetization is shown 
along the bisegmented NW images (longitudinal cross-sections) while the color scale in transverse cross section images corresponds to the axial component of magnetization $m_{x}$. The results show that the Ni segments are mainly axially magnetized along the NW axis (e.g. the fractional axial remanence of $\mathrm{Ni}$ segment magnetization, normalized to its saturation magnetization, is $\left.m_{r}=0.78\right)$. Note the white and light blue-red colors almost everywhere in the longitudinal cross-section as well as a small curling magnetization component extending along the entire $\mathrm{Ni}$ segment, visible also in the transverse cross sections of Figures 4 a.1, b.1, b.2. The magnetization distribution in these transverse cross sections indicate that the curling structures present a large area where $m_{x}$ component is predominant (indicated by red color). The core of all curling structures is displaced from the nanowire axis towards one of the NW sides, following the CoNi magnetization configuration in the adjacent segment of CoNi. Overall, this structure is similar to the vortices with very large core observed reported in FeCo NWs. ${ }^{11}$

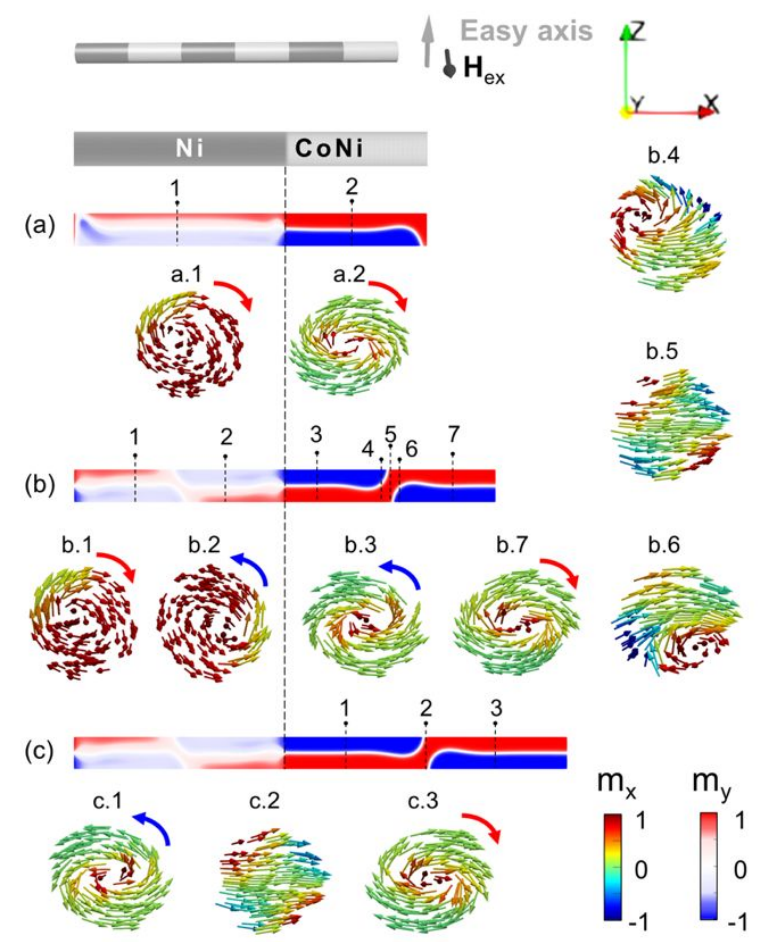

Figure 4. Schematic illustration of the simulated multisegmented NW with indication of the anisotropy easy axis and the perpendicular magnetic field (top panel). Panels $(a, b, c)$, longitudinal cross sections of nanowires showing the magnetization distribution at remanence for various lengths of CoNi sgments (a) $600 \mathrm{~nm}$, (b) $900 \mathrm{~nm}$ and (c) $1200 \mathrm{~nm}$. The axial cross sections are colored according to the transverse component of magnetization, $m_{y}$. The labels 1-7 indicate the positions of the transverse cross-sections a.1-a.2, b.1-b.7 and c.1-c.3. from (a), (b) and (c) 
respectively. The arrows inside the transverse cross-sections represent the local magnetization direction, and they are colored by the axial component of magnetization $\mathrm{m}_{\mathrm{x}}$.

Simulations indicate that the magnetization structure in nanowires with shortest CoNi segments (Figure 4 a.2) consists of a single vortex domain with the same polarity and chirality as the curling component of magnetization in the Ni segments (Figure 4 a.1). We have checked that the magnetization curling indeed starts in the Ni segments. This happens due to the fact that in CoNi the shape anisotropy is counterbalanced by the magnetocrystalline anisotropy, perpendicular to it, and its effect is largely reduced. In $\mathrm{Ni}$ segments separated by short CoNi segments, the curling at the ends occurs with the same rotational sense, probably due to magnetostatic coupling through short CoNi segments, which prefers a parallel chirality configuration. The formation of curling structures in $\mathrm{Ni}$ segments is followed immediately by the magnetization curling in $\mathrm{CoNi}$ segments through the exchange coupling at the interfaces and mimics the curling sense of the $\mathrm{Ni}$ segments, due to the minimization of total energy, i.e. avoiding magnetic charges at the interface between the two materials.

In the case of multisegmented NWs with longer CoNi segments (900 and $1200 \mathrm{~nm}$ ), two curling regions with opposite chirality are formed along the Ni segment as observed in Figures $4(b-c)$, where now the fractional axial remanence of $\mathrm{Ni}$ is $\mathrm{m}_{\mathrm{r}}=0.75$. The longer CoNi segments provide more spacing between $\mathrm{Ni}$ segments which effectively become magnetostatically decoupled. In this case, the curling structures in Ni are formed with opposite chirality as in individual NWs due to magnetic torque effects. ${ }^{41}$ As a consequence of two Ni curling regions, the magnetic domain structure of CoNi segments consists in a pair of vortices with opposite chirality (see Figures 4 (b-c)). The left vortex in this segment represents actually a prolongation of the curling component from the $\mathrm{Ni}$ segment (Figures 4 b.2, b.3). Hence, the whole nanowire shows alternating chirality as a result of the energy minimization (Figures 4 b.1-b.7). The region between two vortex domains is divided by an area with transverse magnetization, which locally minimizes the exchange energy (Figures 4 b.4-b.6) similar to transverse domain states observed in nanotubular systems. ${ }^{42,43}$ For the $3 \mathrm{D}$ representation of the region between two vortex domains see section II(a) of the Supplementary Information. 
The section II of the Supplementary Information compiles the results of modeling under the assumption of the rotation of the c-axis along CoNi segment. In this case, more than two vortex domains are obtained in each segment. Also, additional information is given in section II(c) about the simulations related to different directions of the axial magnetization in $\mathrm{Ni}$ segments which have a small influence on the observed magnetization structures. Thus, the small magnetization curling in the neighboring $\mathrm{Ni}$ segment is a more important factor influencing the structure of CoNi segments than the direction of the large axial Ni magnetization component.

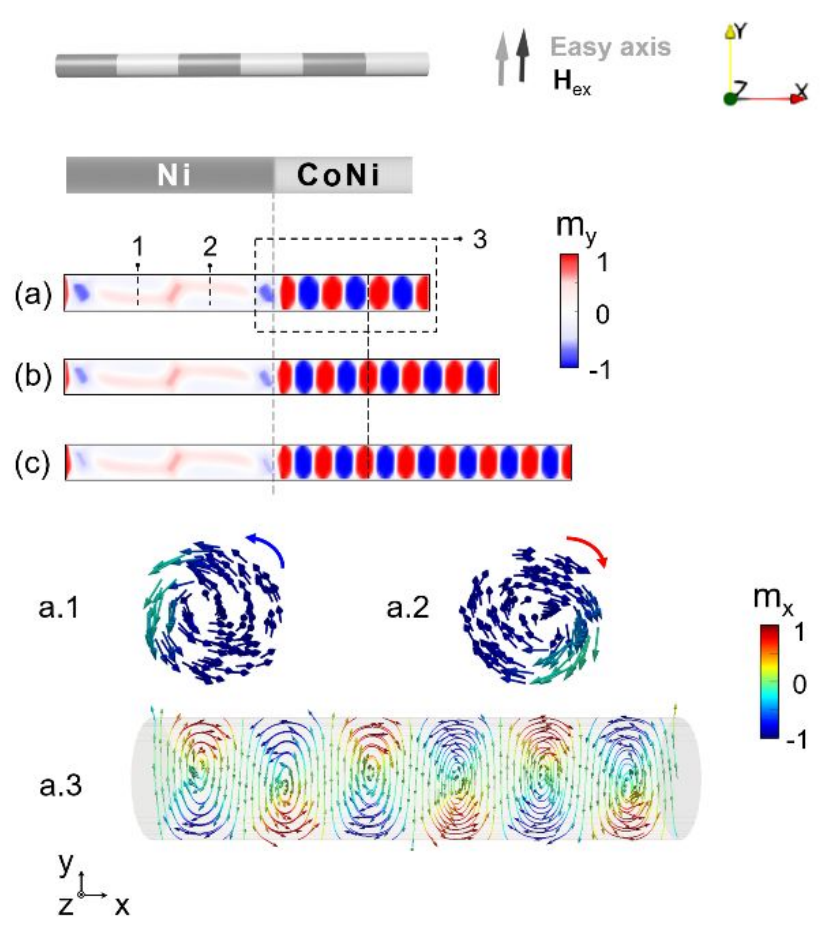

Figure 5. (Top panel) Schematic illustration of the simulated multisegmented NW and indication of the direction of magnetocrystalline anisotropy easy axis and the perpendicular applied magnetic field, $\mathrm{H}_{\mathrm{ex}}$. Panels $(a, b, c)$ indicate longitudinal cross sections showing the magnetization distribution at remanence for various lengths of CoNi sgments (a) $600 \mathrm{~nm}$, (b) $900 \mathrm{~nm}$ and (c) $1200 \mathrm{~nm}$. The axial cross sections are colored according to the transverse component of magnetization, $m_{y}$. The labels 1-3 indicate the positions of the transverse cross-sections a.1-3. The arrows inside the transverse cross-sections represent the local magnetization direction, and they are colored by the axial component of magnetization $m_{x}$. Panel a. 3 shows the magnetization distribution on the nanowire surface in the region enclosed by the dashed square 3 . 
The simulations in Figure 5 correspond to the structure of magnetic domains formed in the CoNi segment at remanence after the application of a magnetic field perpendicular to NW, but parallel to the magnetocrystalline anisotropy easy axis. The domain structure in all of the CoNi segments (various lengths) consists of an ordered sequence of multiple transverse domains where magnetization alternates its direction along the easy axis as previously reported in other nanostructures. ${ }^{21}$ The width of a single transverse domains is $97 \mathrm{~nm}, 89 \mathrm{~nm}$ and $85 \mathrm{~nm}$, respectively for the NWs with $600 \mathrm{~nm}, 900 \mathrm{~nm}$ and $1200 \mathrm{~nm}$ CoNi segments. These values agree with the domain width of about $105 \mathrm{~nm}$ observed in the experiments (See Figure 2 (d1)). Note that the transition between neighboring transverse domains (see Figure 5a.3) occurs by the formation of vortices on the NW surface with cores pointing perpendicular to the NW (Further details provided in section II(a) of the Supplementary Information). These vortices have been recently observed by Electron Holography. ${ }^{29}$

\section{Conclusions}

Multisegmented cylindrical nanowires have been synthesized with designed alternating $\mathrm{CoNi} / \mathrm{Ni}$ composition in order to tailor the magnetic states of individual segments through the effects of materials geometry and anisotropy.

XMCD-PEEEM imaging of multisegmented NWs reveals that the magnetic structure in $\mathrm{Ni}$ segment is in a dominantly axial magnetic state while in CoNi segments it depends on their length. In NWs with the shortest CoNi segments $(600 \mathrm{~nm})$, we observed single vortex domains, in longer segments of $1200 \mathrm{~nm}$ we observed one to three vortex domain states, while in the NWs with the longest CoNi segments either multi-vortex or multitransverse domains were observed.

To disentangle different contributions to the observed behavior, we performed micromagnetic simulations. While one might think that the shape factor of CoNi segments could play the decisive role, we note that the segments are long enough for the difference in shape anisotropy to be minor. Our simulations show that although the $\mathrm{Ni}$ is in an almost single-domain state, it is not completely saturated, and presents a small magnetization curling on the NW surface. Its chirality is formed prior to formation 
of magnetic structures in $\mathrm{CoNi}$ and defines the state of CoNi, that is its chirality along the whole CoNi segment. Here, the magnetostatic coupling of $\mathrm{Ni}$ segments plays a decisive role. For longer CoNi segments, $\mathrm{Ni}$ segments become decoupled, and the curling structures are formed with different chirality at the opposite ends of $\mathrm{Ni}$ segments as in individual NWs. CoNi domains mimics this chirality (at least at the interface CoNi/Ni) in order to avoid magnetic charges at the transition.

On the other hand, the remanent magnetic structure of CoNi segments depends on the direction of the previously applied field. If the direction is perpendicular to both the NW and the magnetization easy axis, the vortex domains simply expand inside the segment following the structures formed at the interfaces and resulting in one vortex domain for short segments and two or more vortex structures for longer segments. The change of easy axis direction along one segment may play here an important role in formation of two or more structures. In turn, when the field is applied perpendicular to the NW but along the magnetization easy axis, transverse domains are formed. They are separated by vortex domain structures with core at the surface pointing perpendicular to it. This state can be alternatively viewed as multiple vortex domains where the vortex cores are expelled to the surface (perpendicular to the anisotropy axis to minimize this energy) and transverse domains are formed in between. In even larger CoNi segments, we should not exclude the influence of defects which can additionally promote the multidomain states.

Our results demonstrate a complex interplay of different energetic contributions and geometry determining the resulting magnetic structures. We stress the importance of different factors which should be taken into account for the design of magnetic NWs. Particularly, we show that an apparently trivial axial magnetization structure in $\mathrm{Ni}$ segments has a strong effect on the neighboring segments through a tiny curling of magnetization on the surface, not even visible in imaging techniques. The overall results show how important the cylindrical geometry of magnetic nanowires is to promote novel physics related to magneto-chiral effects.

\section{Methods/Experimental}


CoNi/Ni nanowires were prepared by electroplating filling the pores of anodic aluminum oxide (AAO) membranes. These templates were obtained by hard anodization in oxalic aqueous solution $(0.3 \mathrm{M})$ containing 5 vol.\% ethanol at a constant temperature of 0 $1^{\circ} \mathrm{C} .{ }^{25,31}$ Pores with $140 \mathrm{~nm}$ in diameter and $60 \mu \mathrm{m}$ in length were obtained. Afterwards, the residual $\mathrm{Al}$ and the alumina barrier layer at the bottom of the foils were chemically etched, and an Au layer was sputtered to serve later as an electrode for final electroplating of the nanowires. The multisegmented nanowires were grown inside the nanopores, at room-temperature, by DC electrodeposition using two different electrolytes $0.75 \mathrm{M} \mathrm{NiSO}_{4}+0.3 \mathrm{M} \mathrm{NiCl}_{2}+0.3 \mathrm{M} \mathrm{H}_{3} \mathrm{BO}_{3}$ and $0.178 \mathrm{M} \mathrm{CoSO}_{4}+0.095 \mathrm{M}$ $\mathrm{NiSO}_{4}+0.32 \mathrm{M} \mathrm{H}_{3} \mathrm{BO}_{3}+0.056 \mathrm{M} \mathrm{C}_{6} \mathrm{H}_{8} \mathrm{O}_{6}$ for different periods of time. Both segments were electrodeposited at a constant voltage of 1.1V (Ni segments) and 1.2 V (CoNi segments) for 35 seconds $\mathrm{Ni}$ and 35, 50 and 60 seconds for CoNi, respectively. The bilayers were repeated 10 times. Finally, the alumina was dissolved by using a mixed solution of $\mathrm{CrO}_{3}$ and $\mathrm{H}_{3} \mathrm{PO}_{4}$. After repeatedly wash of individual nanowires using sonification, they were dispersed on a Si substrate by spin coating.

The XMCD-PEEM measurements were performed at the CIRCE beamline of the ALBA Synchrotron Light Facility (Barcelona, Spain) using an ELMITEC LEEM III instrument with energy analyzer. The samples are illuminated with circularly polarized Xrays at a grazing angle of $16^{\circ}$ with respect to the surface. The emitted photoelectrons (low energy secondary electron with ca. $1 \mathrm{eV}$ kinetic energy) used to form the surface image are proportional to the X-ray absorption coefficient and thus the element-specific magnetic domain configuration is given by the pixel-wise asymmetry of two PEEM images sequentially recorded with left-and right-handed circular polarization (for more details see Supplementary Information).

Micromagnetic modelling of individual multisegmented CoNi-Ni nanowires has been carried out using a finite difference discretization scheme implemented in mumax 3 software. ${ }^{38}$ The simulated nanowire, $140 \mathrm{~nm}$ in diameter, consists of a Ni segment of 900 $\mathrm{nm}$ length and a CoNi segment with periodic boundary conditions. The length of the CoNi segment is set to 600,900 or $1200 \mathrm{~nm}$. The material parameters and the crystal structure are detailed in Table I. The magnetocrystalline easy axis in CoNi is placed nearly 
perpendicular to the nanowire axis. The magnetic remanent state has been evaluated after the application of a magnetic field perpendicular to the nanowire.

\section{ASSOCIATED CONTENT}

\section{Supplementary Information.}

Details and schematic view of XMCD-PEEM contrast, micromagnetic simulations of domain walls between vortices, influence of $\mathrm{Ni}$ axial magnetization and rotation of easy axis in CoNi segments. This material is available free of charge at https://pubs.acs.org.

\section{AUTHOR INFORMATION}

Corresponding Author: Cristina Bran, email: cristina.bran@icmm.csic.es

\section{Acknowledgements}

This investigation has been carried out under the support of Spanish MINECO under project MAT2016-76824-C3-1-R, FIS2016-78591-C3-3-R and the Regional Government of Madrid under project S2018/NMT-4321 NANOMAGCOST-CM. LA and MF acknowledge funding through RTI2018-095303-B-C53. AFR acknowledges support from the Spanish MINECO (MAT2015-68772-P). We also acknowledge the service from the MiNa Laboratory at IMN, and funding from CM (project SpaceTec, S2013/ICE2822), MINECO (project CSIC13-4E-1794) and EU (FEDER, FSE).

\section{References}

(1) Vazquez M. Magnetic Nano- and Microwires, $2^{\text {nd }}$ edition, Elsevier, Amsterdam 2020, Part 3, pp 673-955.

(2) Fernández-Pacheco, A.; Streubel, R.; Fruchart, O.; Hertel, R.; Fischer, P.; Cowburn, R. P. Three-Dimensional Nanomagnetism. Nat. Commun. 2017, 8, 
15756-15769.

(3) Staňo, M.; Fruchart, O. Magnetic Nanowires and Nanotubes. Handb. Magn. Mater. 2018, 27, 155-267.

(4) Charilaou, M.; Braun, H.-B.; Löffler, J. F. Monopole-Induced Emergent Electric Fields in Ferromagnetic Nanowires. Phys. Rev. Lett. 2018, 121, 97202-97206.

(5) Streubel, R.; Fischer, P.; Kronast, F.; Kravchuk, V. P.; Sheka, D. D.; Gaididei, Y.; Schmidt, O. G.; Makarov, D. Magnetism in Curved Geometries. J. Phys. D. Appl. Phys. 2016, 49, 363001-363044.

(6) HERTEL, R. Curvature-Induced Magnetochirality. SPIN 2013, 03, 13400091340017.

(7) Yan, M.; Kákay, A.; Gliga, S.; Hertel, R. Beating the Walker Limit with Massless Domain Walls in Cylindrical Nanowires. Phys. Rev. Lett. 2010, 104, 57201-57204.

(8) Proenca, M. P.; Muñoz, M.; Villaverde, I.; Migliorini, A.; Raposo, V.; Lopez-Diaz, L.; Martinez, E.; Prieto, J. L. Deterministic and Time Resolved Thermo-Magnetic Switching in a Nickel Nanowire. Sci. Rep. 2019, 9-16 .

(9) Salem, M. S.; Sergelius, P.; Corona, R. M.; Escrig, J.; Görlitz, D.; Nielsch, K. Magnetic Properties of Cylindrical Diameter Modulated Ni80Fe20 Nanowires: Interaction and Coercive Fields. Nanoscale 2013, 5, 3941-3947.

(10) Berganza, E.; Bran, C.; Jaafar, M.; Vazquez, M.; Asenjo, A. Domain Wall Pinning in FeCoCu Bamboo-Like Nanowires. Sci. Rep. 2016, 6, 29702-29708.

(11) Bran, C.; Berganza, E.; Palmero, E. M. M.; Fernandez-Roldan, J. A. A.; Del Real, R. P. P.; Aballe, L.; Foerster, M.; Asenjo, A.; Fraile Rodríguez, A.; Vazquez, M. Spin Configuration of Cylindrical Bamboo-Like Magnetic Nanowires. J. Mater. Chem. C 2016, 4, 978-984.

(12) Rodríguez, L. A. A.; Bran, C.; Reyes, D.; Berganza, E.; Vázquez, M.; Gatel, C.; Snoeck, E.; Asenjo, A. Quantitative Nanoscale Magnetic Study of Isolated Diameter-Modulated FeCoCu Nanowires. ACS Nano 2016, 10, 9669-9678.

(13) Méndez, M.; González, S.; Vega, V.; Teixeira, J.; Hernando, B.; Luna, C.; Prida, V. Ni-Co Alloy and Multisegmented Ni/Co Nanowire Arrays Modulated in Composition: Structural Characterization and Magnetic Properties. Crystals 2017, 7, 66-81.

(14) Grutter, A. J.; Krycka, K. L.; Tartakovskaya, E. V; Borchers, J. A.; Reddy, K. S. M.; Ortega, E.; Ponce, A.; Stadler, B. J. H. Complex Three-Dimensional Magnetic Ordering in Segmented Nanowire Arrays. ACS Nano 2017, 11, 8311-8319.

(15) Bran, C.; Berganza, E.; Fernandez-Roldan, J. A.; Palmero, E. M.; Meier, J.; Calle, E.; Jaafar, M.; Foerster, M.; Aballe, L.; Fraile Rodriguez, A.; Chubykalo-Fesenko, 
O.; Vazquez, M. Magnetization Ratchet in Cylindrical Nanowires. ACS Nano 2018, 12, 5932-5939.

(16) Bran, C.; Ivanov, Y. P. P.; Kosel, J.; Chubykalo-Fesenko, O.; Vazquez, M. Co/Au Multisegmented Nanowires: A 3D Array of Magnetostatically Coupled Nanopillars. Nanotechnology 2017, 28, 095709-095715.

(17) Özkale, B.; Shamsudhin, N.; Chatzipirpiridis, G.; Hoop, M.; Gramm, F.; Chen, X.; Martí, X.; Sort, J.; Pellicer, E.; Pané, S. Multisegmented FeCo/Cu Nanowires: Electrosynthesis, Characterization, and Magnetic Control of Biomolecule Desorption. ACS Appl. Mater. Interfaces 2015, 7, 7389-7396.

(18) Ivanov, Y. P.; Chuvilin, A.; Lopatin, S.; Kosel, J. Modulated Magnetic Nanowires for Controlling Domain Wall Motion: Toward 3D Magnetic Memories. ACS Nano 2016, 10, 5326-5332.

(19) Wang; Mukhtar; Wu; Gu; Cao. Multi-Segmented Nanowires: A High Tech Bright Future. Materials, 2019, 12, 3908-3937.

(20) Rippard, W. H.; Pufall, M. R.; Kaka, S.; Silva, T. J.; Russek, S. E.; Katine, J. A. Injection Locking and Phase Control of Spin Transfer Nano-Oscillators. Phys. Rev. Lett. 2005, 95, 067203-067206.

(21) Murapaka, C.; Goolaup, S.; Purnama, I.; Lew, W. S. Coupled Domain Wall Oscillations in Magnetic Cylindrical Nanowires. J. Appl. Phys. 2015, 117, 053913053917.

(22) Pribiag, V. S.; Krivorotov, I. N.; Fuchs, G. D.; Braganca, P. M.; Ozatay, O.; Sankey, J. C.; Ralph, D. C.; Buhrman, R. A. Magnetic Vortex Oscillator Driven by D.C. Spin-Polarized Current. Nat. Phys. 2007, 3 , 498-503.

(23) Abreu Araujo, F.; Piraux, L. Spin-Transfer-Torque Driven Vortex Dynamics in Electrodeposited Nanowire Spin-Valves, Spin 2017, 7, 1740007-1740018.

(24) Franken, J. H.; Swagten, H. J. M.; Koopmans, B. Shift Registers Based on Magnetic Domain Wall Ratchets with Perpendicular Anisotropy. Nat. Nanotechnol. 2012, 7, 499-503.

(25) Berganza, E.; Jaafar, M.; Bran, C.; Fernández-Roldán, J. A. A.; ChubykaloFesenko, O.; Vázquez, M.; Asenjo, A. Multisegmented Nanowires: A Step towards the Control of the Domain Wall Configuration. Sci. Rep. 2017, 7 , 1-8.

(26) Ruiz-Gómez, S.; Foerster, M.; Aballe, L.; Proenca, M. P.; Lucas, I.; Prieto, J. L.; Mascaraque, A.; de la Figuera, J.; Quesada, A.; Pérez, L. Observation of a Topologically Protected State in a Magnetic Domain Wall Stabilized by a Ferromagnetic Chemical Barrier. Sci. Rep. 2018, 8, 16695-16700.

(27) Ivanov, Y. P.; Vázquez, M.; Chubykalo-Fesenko, O. Magnetic Reversal Modes in 
Cylindrical Nanowires. J. Phys. D. Appl. Phys. 2013, 46, 485001-485011.

(28) Ivanov, Y. P.; Trabada, D. G.; Chuvilin, A.; Kosel, J.; Chubykalo-Fesenko, O.; Vázquez, M. Crystallographically Driven Magnetic Behaviour of Arrays of Monocrystalline Co Nanowires. Nanotechnology 2014, 25, 475702-475712.

(29) Andersen, I. M.; Rodríguez, L. A.; Bran, C.; Marcelot, C.; Joulie, S.; Hungria, T.; Vazquez, M.; Gatel, C.; Snoeck, E. Exotic Transverse-Vortex Magnetic Configurations in CoNi Nanowires. ACS Nano 2019, 14, 1399-1405.

(30) Lee, D. J.; Kim, E.; Kim, D.; Park, J.; Hong, S. Nano-Storage Wires. ACS Nano 2013, 7, 6906-6913.

(31) Bran, C.; Fernandez-Roldan, J. A. A.; Palmero, E. M. M.; Berganza, E.; Guzman, J.; Del Real, R. P. P.; Asenjo, A.; Fraile Rodríguez, A.; Foerster, M.; Aballe, L.; Chubykalo-Fesenko, O.; Vazquez, M. Direct Observation of Transverse and Vortex Metastable Magnetic Domains in Cylindrical Nanowires. Phys. Rev. B 2017, 96, 125415-125420.

(32) Palmero, E. M.; Bran, C.; Del Real, R. P.; Vázquez, M. Vortex Domain Wall Propagation in Periodically Modulated Diameter FeCoCu Nanowire as Determined by the Magneto-Optical Kerr Effect. Nanotechnology 2015, 26, $461001-461006$.

(33) Aballe, L.; Foerster, M.; Pellegrin, E.; Nicolas, J.; Ferrer, S. The ALBA Spectroscopic LEEM-PEEM Experimental Station: Layout and Performance. J. Synchrotron Radiat. 2015, 22, 745-752.

(34) Da Col, S.; Jamet, S.; Rougemaille, N.; Locatelli, A.; Mentes, T. O.; Burgos, B. S.; Afid, R.; Darques, M.; Cagnon, L.; Toussaint, J. C.; Fruchart, O. Observation of Bloch-Point Domain Walls in Cylindrical Magnetic Nanowires. Phys. Rev. B 2014, 89, $180405-180410$.

(35) Kimling, J.; Kronast, F.; Martens, S.; Böhnert, T.; Martens, M.; Herrero-Albillos, J.; Tati-Bismaths, L.; Merkt, U.; Nielsch, K.; Meier, G. Photoemission Electron Microscopy of Three-Dimensional Magnetization Configurations in Core-Shell Nanostructures. Phys. Rev. B - Condens. Matter Mater. Phys. 2011, 84, 174406174410.

(36) Schöbitz, M.; De Riz, A.; Martin, S.; Bochmann, S.; Thirion, C.; Vogel, J.; Foerster, M.; Aballe, L.; Menteș, T. O.; Locatelli, A.; Genuzio, F.; Le-Denmat, S.; Cagnon, L.; Toussaint, J. C.; Gusakova, D.; Bachmann, J.; Fruchart, O. Fast Domain Wall Motion Governed by Topology and CErsted Fields in Cylindrical Magnetic Nanowires. Phys. Rev. Lett. 2019, 123, 217201-217205.

(37) Foerster, M.; Prat, J.; Massana, V.; Gonzalez, N.; Fontsere, A.; Molas, B.; Matilla, 
O.; Pellegrin, E.; Aballe, L. Custom Sample Environments at the ALBA XPEEM. Ultramicroscopy 2016, 171, 63-69.

(38) Vansteenkiste, A.; Leliaert, J.; Dvornik, M.; Helsen, M.; Garcia-Sanchez, F.; Van Waeyenberge, B. The Design and Verification of MuMax3. AIP Adv. 2014, 4, 107133-107154.

(39) Kronmüller, H.; Fischer, R.; Hertel, R.; Leineweber, T. Micromagnetism and the Microstructure in Nanocrystalline Materials. J. Magn. Magn. Mater. 1997, 175, 177-192.

(40) Moreno, R.; Evans, R. F. L.; Khmelevskyi, S.; Muñoz, M. C.; Chantrell, R. W.; Chubykalo-Fesenko, O. Temperature-Dependent Exchange Stiffness and Domain Wall Width in Co. Phys. Rev. B 2016, 94, 104433-104438.

(41) Fernandez-Roldan, J. A.; Ivanov, Y. P.; Chubykalo-Fesenko, O. Micromagnetic Modeling of Magnetic Domain Walls and Domains in Cylindrical Nanowires, Magnetic Nano- and Microwires, 2nd edition, Elsevier, Amsterdam, 2020, 403426.

(42) Fernandez-Roldan, J. A.; Chrischon, D.; Dorneles, L. S.; Chubykalo-Fesenko, O.; Vazquez, M.; Bran, C. A Comparative Study of Magnetic Properties of Large Diameter Co Nanowires and Nanotubes. Nanomaterials 2018, 8, 692-703.

(43) Fernandez-Roldan, J. A.; Perez Del Real, R.; Bran, C.; Vazquez, M.; ChubykaloFesenko, O. Magnetization Pinning in Modulated Nanowires: From Topological Protection to the "Corkscrew" Mechanism. Nanoscale 2018, 10, 5923-5927. 